\title{
Production of doubly charmed baryons nearly at rest
}

\author{
Stefan Groote ${ }^{a}$, Sergey Koshkarev \\ Institute of Physics, University of Tartu, Tartu 51010, Estonia
}

Received: 16 June 2017 / Accepted: 18 July 2017 / Published online: 1 August 2017

(C) The Author(s) 2017. This article is an open access publication

\begin{abstract}
We investigate the production cross sections, momentum distributions and rapidity distributions for doubly charmed baryons which according to the intrinsic heavy quark mechanism are produced nearly at rest. These events should be measurable at fixed-target experiments like STAR@RHIC and AFTER@LHC.
\end{abstract}

\section{Introduction}

Doubly heavy baryons are a rigorous prediction of quantum chromodynamics. In the 2000s, evidence for the existence of the doubly charmed baryons was reported by the SELEX Collaboration [1-6]. However, the discrepancy of the production properties derived from the SELEX data with predictions of perturbative QCD induced wrong expectations for the production rates of the doubly charmed baryons in the different production environments, leading to reports stating the nonevidence of doubly heavy baryons [7-10].

In Ref. [11] it is shown that the intrinsic charm mechanism can fill the gap between the rates motivated by the SELEX data and the theoretically predicted production rates. Moreover, the ratio $\sigma(c \bar{c} c \bar{c}) / \sigma(c \bar{c})$ calculated from the SELEX data is comparable or even smaller than the same rate derived from the double $J / \psi$ production data at the NA3 experiment $[12,13]$. In addition, the intrinsic charm mechanism can perfectly explain the kinematics of the doubly charmed baryons with $\left\langle x_{F}\right\rangle \sim 0.33$ and the relatively small mean transverse momentum of approx. $1 \mathrm{GeV} / \mathrm{c}$.

The most recent Belle and $\mathrm{LHCb}$ results cannot argue against the SELEX data. The Belle experiment [9] presented an upper limit on the cross section $\sigma\left(e^{+} e^{-} \rightarrow \Xi_{c c}^{+}+X\right)$ of $82-500 \mathrm{fb}$ at $95 \%$ confidence level for the decay mode with $\Lambda_{c}^{+}$at $\sqrt{s}=10.58 \mathrm{GeV}$, using a luminosity of $980 \mathrm{fb}^{-1}$. This turns out to be at least twice as much as the theoretical upper limit given by $\sigma\left(\Xi_{c c}^{+}\right) \approx 35 \pm 10 \mathrm{fb}[14,15]$.

\footnotetext{
a e-mail: groote@ut.ee
}

The LHCb Collaboration [10] published upper limits at 95\% confidence level on the ratio $\sigma\left(\Xi_{c c}^{+}\right) \cdot \operatorname{Br}\left(\Xi_{c c}^{+} \rightarrow\right.$ $\left.\Lambda_{c}^{+} K^{-} \pi^{+}\right) / \sigma\left(\Lambda_{c}^{+}\right)$of $1.5 \times 10^{-2}$ and $3.9 \times 10^{-4}$ for the lifetimes of $100 \mathrm{fs}$ and $400 \mathrm{fs}$, respectively, and for an integrated luminosity of $0.65 \mathrm{fb}^{-1}$. This is comparable with results from Refs. [14,16-18] of about $10^{-4}-10^{-3}$. However, the minimum lifetime reached by the $\mathrm{LHCb}$ is about three times larger than the one measured by the SELEX experiment which is $\tau\left(\Xi_{c c}^{+}\right)<33$ fs at $90 \%$ confidence level [1], and almost two times larger than the theoretical prediction of $\tau\left(\Xi_{c c}^{+}\right) \approx 53$ fs [19]. In other words, the LHCb Collaboration provided an analysis outside the signal region.

The production of doubly heavy baryons at high Feynman$x$ at the scheduled future fixed-target experiment at the LHC (AFTER@LHC) via the intrinsic heavy quark mechanism is already discussed in Refs. [11,20]. In addition, some existing experiments have fixed-target programs [21-23]. However, as these experiments were built as collider detectors, they have very limited access to high Feynman- $x$.

In a recent talk [24], Stanley Brodsky proposed the production of charmed hadrons via the intrinsic charm mechanism from the target (see also chapter 6.1 in Ref [25]). In this paper we investigate the opportunity to produce doubly charmed baryons via the intrinsic heavy quark mechanism from the target at the fixed-target experiment at STAR [21], the fixed-target detector at the LHCb experiment (SMOG@LHCb) $[22,23]$ and at the scheduled future fixedtarget experiment at the LHC (AFTER @LHC) [26,27].

\section{Revisiting the SELEX data}

The SELEX experiment was a fixed-target experiment utilizing the Fermilab negative and positive charged beams at $600 \mathrm{GeV} / \mathrm{c}$ to produce charm particles in a set of thin foil of $\mathrm{Cu}$ or in a diamond. It was operated in the kinematic region $x_{F}>0.1$. The negative beam composition was about $50 \%$ $\Sigma^{-}$and $50 \% \pi^{-}$while the positive beam was composed of 
$90 \%$ protons. The experimental data recorded used both positive and negative beams. $67 \%$ of the events were induced by $\Sigma^{-}, 13 \%$ by $\pi^{-}$, and $18 \%$ by protons.

The production cross section was not provided by the SELEX Collaboration. However, the production properties of the doubly charmed baryons can be compared to that of the $\Lambda_{c}^{+}$baryon. To simplify the analysis we will take a look only at the production of the 20 signal events for the decay mode $\Xi_{c c}^{++} \rightarrow \Lambda_{c}^{+} K^{-} \pi^{+} \pi^{+}$at a mass of $3.76 \mathrm{GeV}$ over a sample of 1656 events for $\Lambda_{c}^{+} \rightarrow p K^{-} \pi^{+}$[2] with $x_{F}\left(\Lambda_{c}^{+}\right)>0.15$. This sample was previously used for a precision measurement of the lifetime of $\Lambda_{c}^{+}[28,29]$. The measured production ratio $R$ is defined by

$$
\begin{aligned}
R & =\frac{\sigma\left(\Xi_{c c}^{++}\right) \cdot \operatorname{Br}\left(\Xi_{c c}^{++} \rightarrow \Lambda_{c}^{+} K^{-} \pi^{+} \pi^{+}\right)}{\sigma\left(\Lambda_{c}^{+}\right)} \\
& =\frac{N_{\Xi_{c c}^{++}}}{\varepsilon_{++}} \cdot \frac{\varepsilon_{\Lambda_{c}^{+}}}{N_{\Lambda_{c}^{+}}}
\end{aligned}
$$

where $N$ is the number of events in the respective sample and the reconstruction efficiency of $\Xi_{c c}^{++}$is given by $1 / \varepsilon_{++} \simeq 3.7$ [2]. Central values for the number $N_{\Lambda_{c}^{+}} / \varepsilon_{\Lambda_{c}^{+}}$ of corrected events can be found in Ref. [30] to lie between 13326 and 10010 according whether the lowest bin with $x_{F} \in[0.125,0.175]$ is taken into account or not. However, using the intrinsic charm mechanism as the production mechanism, $\varepsilon_{++}$will be at least 2.3 times bigger (see Figs. 1 and 2 for the acceptance for perturbative QCD and intrinsic charm, respectively). Therefore, we obtain

$R \sim(2-3) \times 10^{-3}$

This value is at least an order of magnitude smaller than the production ratio in the sample $N_{\Xi_{c c}^{++}} / \varepsilon_{++} \cdot 1 / N_{\Lambda_{c}^{+}} \approx 0.045$, which is usually erroneously used as the production ratio.

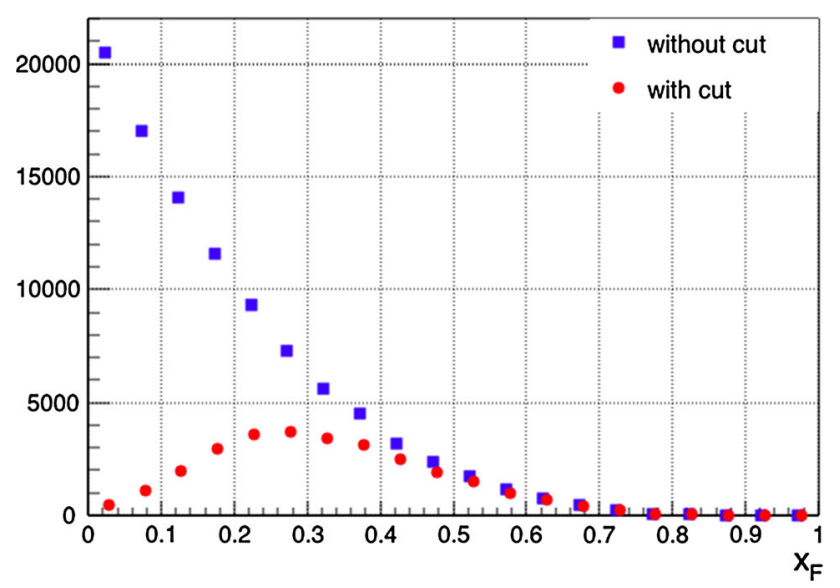

Fig. 1 The squared (blue) points represent the pQCD motivated $x_{F}$ distribution of $\Xi_{c c}^{++}$baryons. The circular (red) points show this distribution with the experimental geometry cut (cf. Ref. [2])

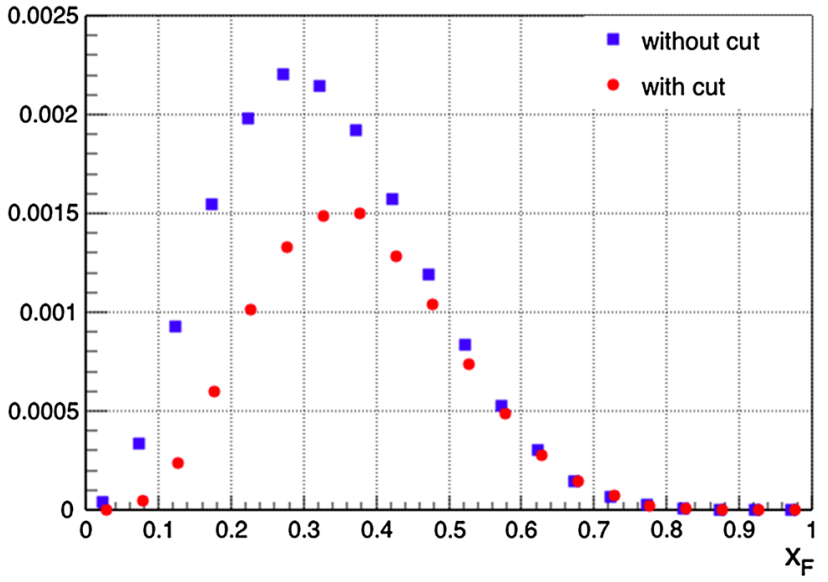

Fig. 2 The squared points represent the IC motivated $x_{F}$ distribution of $\Xi_{c c}^{++}$baryons. The circular point show this distribution re-weighted with the experimental reconstruction efficiency as a function of $x_{F}$. The reconstruction efficiency is extracted from Fig. 1

\section{Theoretical background}

Origin, properties and possibilities for the detection of a non-perturbative intrinsic heavy flavor component in the nucleon are widely discussed in the literature [26,27,31-37]. QCD predicts such components from the outset. Intrinsic charm and bottom quarks are contained in the wave function of a light hadron and originate from diagrams where the heavy quarks are multiply attached via gluons to the valence quarks. Intrinsic heavy flavor components are contributed by the twist-six contribution of the operator product expansion proportional to $1 / m_{Q}^{2}[31,32]$. In this case, the frame-independent light-front wave function of the light hadron has maximum probability if the Fock state is minimally off-shell. This means that all the constituents are at rest in the hadron rest frame and thus have the same rapidity $y$ if the hadron is boosted. Equal rapidity occurs if the light-front momentum fractions $x=k^{+} / P^{+}$of the Fock state constituents are proportional to their transverse masses, $x_{i} \propto m_{T, i}=\left(m_{i}^{2}+k_{T, i}^{2}\right)^{1 / 2}$, i.e. if the heavy constituents have the largest momentum fractions. This features the BHPS model given by Brodsky, Hoyer, Peterson and Sakai for the distribution of intrinsic heavy quarks $[33,34]$.

In the BHPS model the wave function of a hadron in QCD can be represented as a superposition of Fock state fluctuations, e.g. $|h\rangle \sim\left|h_{l}\right\rangle+\left|h_{l} g\right\rangle+\left|h_{l} Q \bar{Q}\right\rangle \ldots$, where $h_{l}$ is the light quark content, and $Q=c, b$. If the projectile interacts with the target, the coherence of the Fock components is broken and the fluctuation can hadronize. The intrinsic heavy flavor Fock components are generated by virtual interactions such as $g g \rightarrow Q \bar{Q}$ where the gluons couple to two or more valence quarks of the projectile. The probability to produce such $Q \bar{Q}$ fluctuations scales as $\alpha_{s}^{2}\left(m_{Q}^{2}\right) / m_{Q}^{2}$ relative to the leading-twist production. 
Following Refs. [33-35], the general formula for the probability distribution of two heavy quark pairs by intrinsic heavy flavor Fock state as a function of the momentum fractions $x_{i}$ is given by

$$
\frac{\mathrm{d} P_{i Q_{1} Q_{2}}}{\prod_{i=1}^{n} d x_{i}} \propto \alpha_{s}^{4}\left(M_{Q_{1} \bar{Q}_{1}}\right) \alpha_{s}^{4}\left(M_{Q_{2} \bar{Q}_{2}}\right) \frac{\delta\left(1-\sum_{i=1}^{n} x_{i}\right)}{\left(\sum_{i=1}^{n} \hat{m}_{T, i}^{2} / x_{i}\right)^{2}}
$$

where $\hat{m}_{i}=\left(m_{i}^{2}+\left\langle k_{T, i}^{2}\right\rangle\right)^{1 / 2}$ is the effective mass, $k_{T, i}^{2}$ is the mean transverse momenta, and the masses of the light quarks are neglected.

The normalization of the production cross section of two charm pairs is given by

$\sigma_{i c c}=\frac{P_{i c c}}{P_{i c}} \cdot \sigma_{i c}, \quad \sigma_{i c}=P_{i c} \cdot \sigma^{\text {inel }} \frac{\mu^{2}}{4 \hat{m}_{c}^{2}}$,

where $\mu^{2} \approx 0.2 \mathrm{GeV}^{2}$ denotes the squared soft interaction scale parameter, the effective transverse $c$-quark mass is given by $\hat{m}_{c}=1.5 \mathrm{GeV}$, and $\sigma^{\text {inel }}$ is the inelastic protonproton cross section.

The nuclear dependence scaling from the manifestation of intrinsic charm is expected to be $\sigma_{A} \approx \sigma_{i c c} \cdot A^{2 / 3}$ for production from the beam and $\sigma_{A} \approx \sigma_{i c c} \cdot A$ for production from the target [24].

\section{Production of the doubly charmed baryons}

The production cross section of the doubly charmed baryon can be obtained as an application of the principle of quarkhadron duality. According to this principle, the cross section of the baryon is obtained by calculating the production of a $Q Q$ pair in the small invariant mass interval between $2 m_{Q}$ and the threshold to produce open heavy quark hadrons, $2 m_{H}$. The $Q Q$ pair has $3 \times 3=(\overline{3}+6)$ color components, consisting of a color antitriplet and a color sextet. The probability that a $Q Q$ pair forms an antitriplet state is $\overline{3} /(\overline{3}+6)=1 / 3$. Therefore, in case of the doubly charmed baryon the cross section will be

$\sigma(c c)=\frac{1}{3} f_{c c} \sigma_{i c c}$

where $f_{c c}$ is the fragmentation ratio of the $c c$ pair written as

$f_{c c}^{i c c}=\int_{4 m_{c}^{2}}^{4 m_{D}^{2}} \mathrm{~d} M_{c c}^{2} \frac{\mathrm{d} P_{i c c}}{\mathrm{~d} M_{c c}^{2}} / \int_{4 m_{c}^{2}}^{s} \mathrm{~d} M_{c c}^{2} \frac{\mathrm{d} P_{i c c}}{\mathrm{~d} M_{c c}^{2}}$.

Not all $c c$ pairs form a doubly charmed baryon. Unfortunately, the fragmentation rate is unknown. However, if we consider the diquark $c c$ as heavy antiquark and analyze the fragmentation of $c$ and $b$ quarks into mesons, for example $f\left(c \rightarrow D^{+}\right)=0.217 \pm 0.043[38]$ or $f\left(b \rightarrow B^{+}, B^{0}\right)=$ $0.344 \pm 0.021$ [39], we can assume the doubly charmed baryonic cross section to be

$\sigma\left(\Xi_{c c}\right) \sim(0.2-0.3) \cdot \sigma(c c)$

The STAR fixed-target program is a fixed-target experiment using the proton beam of the Relativistic Heavy Ion Collider (RHIC) up to $250 \mathrm{GeV} / \mathrm{c}$ and the $\mathrm{Au}$ beam up to $100 \mathrm{GeV} / \mathrm{c}$ colliding with a wired target. Combining Eqs. (2), (3) and (4) and using $\sigma_{p p}^{\text {inel }}\left(p_{\text {beam }}=200 \mathrm{GeV}\right) \approx 32 \mathrm{mb}$ [40], we may expect the production cross section of the $\Xi_{c c}$ to be

$\sigma\left(\Xi_{c c}\right) \approx(0.2-0.3) \times 75 \mathrm{nb}$

The kinematic limits on the energy and the momentum of the doubly charmed baryon formed by the intrinsic charm from the target are given by

$E_{l a b}=\frac{1}{2 m_{\mathrm{tar}}}\left(m_{c c}^{2}+m_{\mathrm{tar}}^{2}\right), \quad p_{l a b}=\frac{1}{2 m_{\mathrm{tar}}}\left(m_{c c}^{2}-m_{\mathrm{tar}}^{2}\right)$.

These last expressions depend solely on the two masses $m_{c c}$ and $m_{\text {tar }}$ and no longer on the beam energy. Upon combining Eqs. (1) and (6) we can find the momentum distribution (Fig. 3) and the distribution of the rapidity difference $\Delta y=y-y_{\operatorname{tar}}$ (Fig. 4) in the laboratory frame. It is obvious that experiments at the STAR detector, typical at rapidities $|y|<1$ for track selection, have the potential to observe doubly charmed baryons.

The SMOG@LHCb is a fixed-target experiment using the LHC beam at $6500 \mathrm{GeV} / \mathrm{c}$ dumped in the helium gas target. Following the logic from above and using $\sigma_{p p}^{\text {inel }}(\sqrt{s}=$ $110 \mathrm{GeV}) \approx 37 \mathrm{mb}$ [41] we obtain the production cross section of the $\Xi_{c c}$ to be

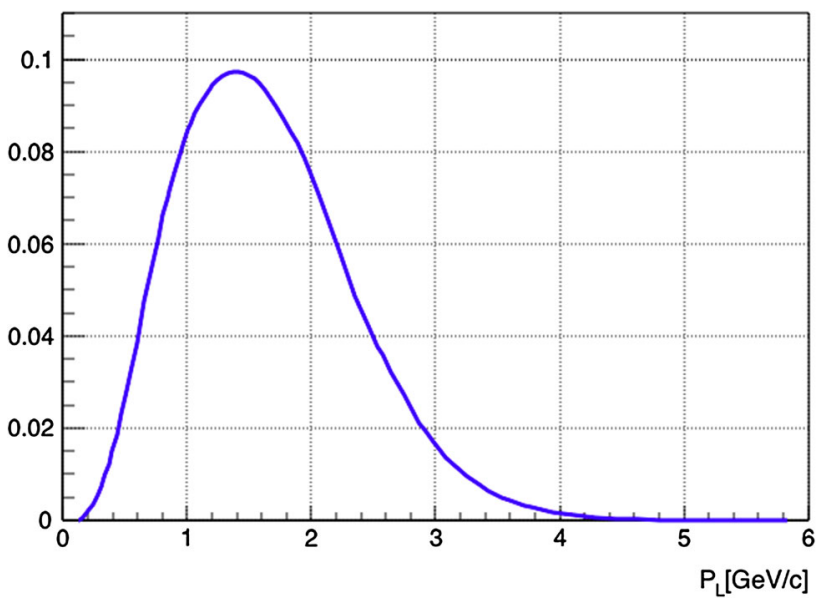

Fig. 3 Momentum distribution of the $\Xi_{c c}$ baryons produced by the intrinsic charm from the target with a $200 \mathrm{GeV} / \mathrm{c}$ proton beam 


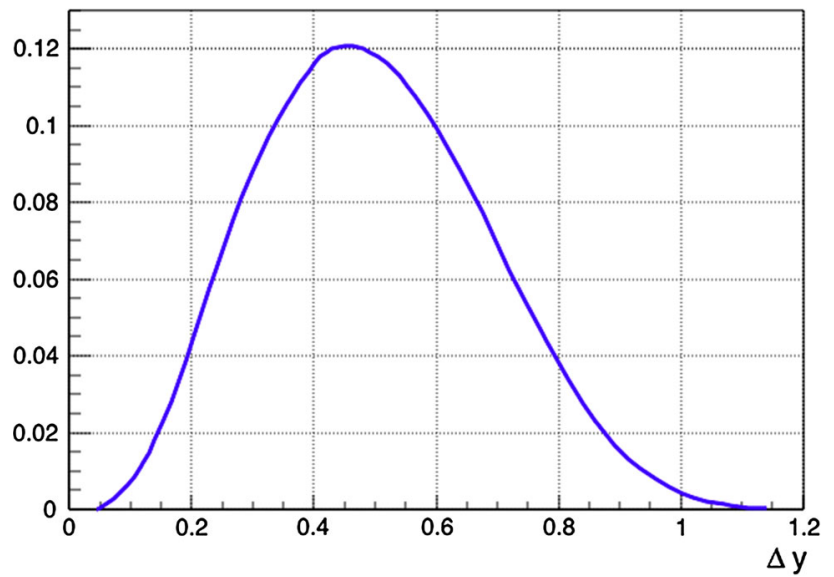

Fig. 4 Rapidity difference of the $\Xi_{c c}$ baryons produced by the intrinsic charm from the target with a $200 \mathrm{GeV} / \mathrm{c}$ proton beam

$\sigma\left(\Xi_{c c}\right) \approx(0.2-0.3) \times 65 \mathrm{nb}$.

The kinematic distributions have the same shape with slightly different mean values. Unfortunately, SMOG@LHCb has acceptance only for $\Delta y>2$ which will make the detection of the doubly charmed baryons problematic.

AFTER@LHC is the scheduled future fixed-target experiment at the LHC operating at $\sqrt{s}=115 \mathrm{GeV}$. Therefore, the estimation provided for SMOG@LHCb can also be used forAFTER@LHC.

\section{Conclusion}

In this paper we investigated the beautiful prediction of the intrinsic heavy quark mechanism. The doubly charmed baryons are produced from the target with an approximate mean value for the momentum of about $1.5 \mathrm{GeV} / \mathrm{c}$. Such "soft" final states can be observed at the current and future fixed-target experiments. The production cross sections are presented.

From the calculation we see that the intrinsic heavy quark mechanism does not contribute to the region of negative $x_{F}$. The double intrinsic heavy quark mechanism is not the leading production mechanism at the modern accelerators [42]. However, it still can aim to searching exotic states like double charmed baryons. In addition, we reinterpreted the SELEX data and obtained a realistic production ratio for doubly charmed baryons $(\mathrm{ccu})$ over $\Lambda_{c}^{+}$in the kinematic region of SELEX (see the discussion in Sect. 2).

In the end it is interesting to note that using the fragmentation ratios of $c$ and $b$ quarks into baryons, $f(c \rightarrow$ $\left.\Lambda_{c}^{+}\right)=0.071 \pm 0.21$ [43] and $f(b \rightarrow b$-baryons $)=$ $0.197 \pm 0.046$ [39] we can also roughly calculate the doubly, hidden and open charm, tetraquark production cross section as $0.1 \cdot \sigma(c c)$.
Acknowledgements We would like to thank S.J. Brodsky for very detailed and productive discussions on the production of heavy quark states from the target via the intrinsic heavy quark mechanism. In addition, we would like to thank Guannan Xie for comments on acceptance of the STAR detector and Yu. Shcheglov for comments on the acceptance of the LHCb detector. This work was supported by the Estonian Research Council under Grant No. IUT2-27.

Open Access This article is distributed under the terms of the Creative Commons Attribution 4.0 International License (http://creativecomm ons.org/licenses/by/4.0/), which permits unrestricted use, distribution, and reproduction in any medium, provided you give appropriate credit to the original author(s) and the source, provide a link to the Creative Commons license, and indicate if changes were made. Funded by SCOAP ${ }^{3}$.

\section{References}

1. M. Mattson et al., SELEX Collaboration. Phys. Rev. Lett. 89, 112001 (2002)

2. M. Mattson, Ph.D. thesis (Carnegie Mellon University, 2002)

3. M.A. Moinester et al., SELEX collaboration. Czech. J. Phys. 53, B201 (2003)

4. A. Ocherashvili et al., SELEX Collaboration. Phys. Lett. B 628, 18 (2005)

5. J. Engelfried., SELEX collaboration. Nucl. Phys. A 752, 121 (2005)

6. J. Engelfried., SELEX Collaboration, Proceedings of the International Conference on Heavy Quarks and Leptons (HQL 06), Munich, Germany, 16-20 October 2006, eConf C 0610161, 003 (2006)

7. S.P. Ratti, Nucl. Phys. Proc. Suppl. 115, 33 (2003)

8. B. Aubert et al., BaBar collaboration. Phys. Rev. D 74, 111103 (2006)

9. Y. Kato et al., Belle Collaboration. Phys. Rev. D 89(5), 052003 (2014)

10. R. Aaij et al., LHCb collaboration. JHEP 1312, 090 (2013)

11. S. Koshkarev, V. Anikeev, Phys. Lett. B 765, 171 (2017)

12. J. Badier et al., NA3 collaboration. Phys. Lett. B 114, 457 (1982)

13. J. Badier et al., NA3 collaboration. Phys. Lett. B 158, 85 (1985)

14. V.V. Kiselev and A.K. Likhoded, Phys. Usp. 45, 455 (2002) [Usp. Fiz. Nauk 172, 497 (2002)]

15. V.V. Kiselev, A.K. Likhoded, M.V. Shevlyagin, Phys. Lett. B 332, 411 (1994)

16. C.H. Chang, C.F. Qiao, J.X. Wang, X.G. Wu, Phys. Rev. D 73, $094022(2006)$

17. C.H. Chang, J.P. Ma, C.F. Qiao, X.G. Wu, J. Phys. G34, 845 (2007)

18. D.A. Günter, V.A. Saleev, Phys. Rev. D 64, 034006 (2001)

19. M. Karliner, J.L. Rosner, Phys. Rev. D 90(9), 094007 (2014)

20. S. Koshkarev, Act. Phys. Pol. B 48(2), 163 (2017)

21. K.C. Meehan., STAR Collaboration. J. Phys. Conf. Ser. 742(1), 012022 (2016)

22. R. Aaij et al., LHCb collaboration. JINST 7, P01010 (2012)

23. R. Aaij et al., LHCb collaboration. Int. J. Mod. Phys. A 30(07), $1530022(2015)$

24. S.J. Brodsky, Workshop on LHCb Heavy Ion and Fixed Target physics, CERN, January 9-10 (2017)

25. S.J. Brodsky, V.A. Bednyakov, G.I. Lykasov, J. Smiesko, S. Tokar, Prog. Part. Nucl. Phys. 93, 108 (2017)

26. S.J. Brodsky, F. Fleuret, C. Hadjidakis, J.P. Lansberg, Phys. Rept. 522, 239 (2013)

27. J.P. Lansberg, S.J. Brodsky, F. Fleuret, C. Hadjidakis, Few Body Syst. 53, 11 (2012) 
28. A. Kushnirenko et al., SELEX collaboration. Phys. Rev. Lett. 86, $5243(2001)$

29. A. Kushnirenko, Ph.D. thesis (Carnegie Mellon University, 2000)

30. F.G. Garcia et al., SELEX collaboration. Phys. Lett. B 528, 49 (2002)

31. S.J. Brodsky, J.C. Collins, S.D. Ellis, J.F. Gunion and A.H. Mueller, DOE/ER/40048-21 P4, SLAC-PUB-15471

32. M. Franz, M.V. Polyakov, K. Goeke, Phys. Rev. D 62, 074024 (2000)

33. S.J. Brodsky, P. Hoyer, C. Peterson, N. Sakai, Phys. Lett. B 93, 451 (1980)

34. S.J. Brodsky, C. Peterson, N. Sakai, Phys. Rev. D 23, 2745 (1981)

35. R. Vogt, S.J. Brodsky, Phys. Lett. B 349, 569 (1995)

36. A. Rakotozafindrabe et al., PoS DIS 2013, 250 (2013)
37. S.J. Brodsky, A. Kusina, F. Lyonnet, I. Schienbein, H. Spiesberger, R. Vogt, Adv. High Energy Phys. 2015, 231547 (2015)

38. S. Checkanov et al., ZEUS collaboration. Eur. Phys. J C 44, 351366 (2005)

39. C. Patrignani et al., Particle data group. Chin. Phys. C 40, 100001 (2016)

40. A. Schiz et al., Phys. Rev. D 24, 26 (1981)

41. M.M. Block, F. Halzen, Phys. Rev. D 86, 014006 (2012)

42. S. Koshkarev, S. Groote, Nucl. Phys. B 915, 384 (2017)

43. B. Aubert et al., BaBar collaboration. Phys. Rev. D 75, 012003 (2007) 